\title{
RBFNN based Terminal Sliding Mode Adaptive Control for Electric Ground Vehicles after Tire Blowout on Expressway
}

\author{
Lu Yang ${ }^{a}$, Ming Yue ${ }^{a, b, *}$, Yuanchang $\mathrm{Liu}^{c}$, Lie Guo $^{a}$ \\ ${ }^{a}$ School of Automotive Engineering, Dalian University of Technology, Dalian 116024, China \\ ${ }^{b}$ State Key Laboratory of Robotics and System, Harbin Institute of Technology, Harbin 150001, China \\ ${ }^{c}$ Department of Mechanical Engineering, University College London, London WC1E7JE, UK
}

\begin{abstract}
This paper proposes a radial basis function neural network (RBFNN) based terminal sliding mode control scheme for electric ground vehicles subject to tire blowout on expressway in presence of tire nonlinearities, unmodeled dynamics and external disturbances. For enhancing the longitudinal and lateral stability of the vehicle after tire blowout, a saturated velocity planner is firstly constructed for tracking the original motion trajectory, by which the longitudinal velocity and yaw rate saturation constraints can be effectively handled. Afterwards, a terminal sliding mode controller (TSMC) is designed for tracking the planned velocity signals because of its inherent finite time convergence rate and superior steady-state property, by which the adverse dynamic behaviors can be timely suppressed. Further, to strengthen the adaptability and robustness of the control scheme, a RBFNN approximator is developed for identifying the lumped uncertainty, such as tire nonlinearities, unmodeled dynamics and external disturbances, etc., and then compensated into the controller. Lastly, simulations with front-right tire blowout on expressway are performed to validate the effectiveness and efficiency of presented control scheme and methods, and the comprehensive performance of TSMC+RBFNN and TSMC schemes in maintaining original trajectory tracking capacity is evaluated and discussed.
\end{abstract}

Keywords: Lumped uncertainties, Saturated velocity planning, Terminal sliding mode control, Tire blowout, Radial basis function neural network

${ }^{*}$ Corresponding author

Preprint submitted to Applied Soft Computing

April 14, 2020 


\section{Introduction}

\subsection{Background and Motivations}

Tire, as the only component between vehicle and ground, is crucial to achieving the predefined performance of the vehicle through the drive, brake and steering maneuvers [1]. Inevitably, tire blowout may happen due to the inappropriate usage, such as too high or too low tire pressure, tire wear, and overload etc., which will leads to the vehicle instability and terrible accidents. As reported in [2], the proportion of accidents caused by tire blowout in expressway accidents is $32 \%$, in which the death rate is close to $49 \%$; while the death rate has reached $80 \%$ when the speed is $120 \mathrm{~km} / \mathrm{h}$. Therefore, tire blowout on expressway is regarded as one of the main reasons that deduced the serious traffic accidents. Despite most of the vehicle have been equipped with the tire pressure monitoring systems, the system only plays the role of warning the driver, which cannot assist driver to manipulate the vehicle. To address this problem, more and more attention has been paid to investigation on active control for the vehicle after tire blowout $[3,4,5]$. Meanwhile, electric ground vehicles and hybrid electric ground vehicles, particularly for those driven independently by in-wheel motors, have received extensive concern due to fast response, high-energy efficiency and favourable maneuverability [6, 7]. Due to the significantly increase of unsprung masses caused by the in-wheel motors, the electric ground vehicles with tire blowout is more fatal than that in conventional vehicles caused by the drastic tire vertical force change. Hence, tire blow-out control for the electric ground vehicles is a meaningful and challenging study, which motivate us to make a further investigation.

Recent research reveals that the vehicle after tire blowout on expressway will rapidly drift off the original lane because of the instantaneous changed tire mechanical properties [8]. Limited by longitudinal inter-vehicular space and ego vehicle stability constraints, the motion velocities of the vehicle after tire blowout, especially longitudinal velocity and yaw rate, ought to be smoothly constrained within a certain range for preventing vehicular rear-end collision and ego vehicle instability. In addition, the developed control system should have fine convergence time and strong robustness such that the adverse dynamic behaviors can be timely suppressed even in presence of system lumped uncertainties, such as tire nonlinearities, unmodeled dynamics, external disturbances. Under such background, saturated velocity planning [9, 10], terminal sliding mode control (TSMC) $[11,12,13]$, and neural networks approximation $[14,15,16]$ have entered our view due to their excellent constraint treatment, inherent finite-time convergence 
rate and powerful approaching capacity. To this end, this paper attempts to propose an adaptive control scheme for the vehicle after tire blowout via saturated velocity planning, TSMC tracking as well as uncertainty approximation. To the best of our knowledge, there exist no study on the coordinated control for the vehicle after tire blowout considering planning, control and approximation, which motivates us for this work.

\subsection{Literature Review}

Recent research on tire blowout can be divided into two categories: finite-element method (FEM) based tire structure improvements and vehicle motion control after tire blowout. In [17], a structural shell based tire model with materialized sidewall modelling (bead to bead) was constructed, and then the stability of the vehicle with standard tire and run-flat tire was discussed. In [18], the influence of tire blowout on the collision of a light pickup truck with a guardrail safety barrier was studied through finite-element simulation. In [19], the finite element tyre model was established, and the tyre blow-out experiment in the static state validated the effectiveness. To sum up, tire mechanical property during tire blowout are studied with FEM in these literature, which provide preliminary outcomes for mechanical performance improvement so as to prevent tire blow-out. Despite the tire pressure monitoring systems have been equipped with most of the vehicle along with the practice of relevant laws and regulations [20], these only play the role of warning driver and cannot prevent tire blowout. To this end, more and more researchers have devoted to designing and developing the active control method for the vehicle after tire blowout based on the acquired tire pressure information [21, 22].

To implement the active control method, a reliable simulation plant is essential to explore and develop, for example, in [23], a reconfigurable control structure, including of a emergency controller, a nominal controller and feedforward term, was proposed, and tire burst experiment verified the performance of the burst controller. In [24], the Engineering Dynamics Vehicle Simulation Model (EDVSM) was developed for modeling and simulating the transient effects of vehicle after tire blowout, and experiment results confirmed the effectiveness of the tire blowout model in EDVSM. To further improve the simulation plant accuracy, a tire blowout model for a tractor semitrailer in TruckSim-TM was developed in [25], and the experimental data from a left steer axle tire blowout of a tractor trailer was considered for modeling. As well, a CarSim-Simulink co-simulation platform was developed in [4], and an adaptive fuzzy proportional-integral-derivative (PID) control method was presented for maintaining the driving 
stability of the vehicle with tire blowout. Recently, in [26], a new tire blowout model is developed with explicit consideration of tire vertical load redistribution and self-alignment torque, which further improved the simulation accuracy.

In addition to the simulation platform, tire blowout oriented advanced control algorithm design is becoming particularly urgent, which not only has the considerable robustness and adaptability but also could deal with the various physical constraints. In [27], a predictive safety control method for the road vehicle after tire blowout was designed by introducing driving assistant system, which provided a lateral stability control framework for the vehicle with tire blowout. In [28], a gain-scheduling $H_{\infty}$ control approach was proposed to enhance vehicle stability with respect to time-varying vehicle velocity as well as uncertain tire cornering, and the simulation results demonstrated the effectiveness of control approach. These studies greatly improve vehicle stability with tire blowout through the advanced control algorithm, such as predictive control, robust control, etc. Nevertheless, for the vehicle with tire blowout, the velocity of vehicle after tire blowout must be restrained within a certain range so as to prevent the bead separating from the rim [29]. Thus, the vehicle velocity constraints, especially longitudinal velocity and yaw rate, should be constrained within the threshold values while guaranteing the vehicle driving safety. Lately, saturated control method has been used for realizing trajectory tracking of mobile robots under the control input constraints $[9,10]$. In this event, this paper, integrating the system dynamics constraints, proposes a saturated velocity planner for such a vehicle to achieve the system stabilization and original path tracking.

Besides, an effective tracking scheme needs to be developed considering system requirements, such as rapid convergence, strong robustness and acceptable calculation burden. Compared to other methods, sliding mode control (SMC) has attractive superiorities of robustness to overcome uncertainties and disturbance as well as insensitivity to the system parametric variations. In [30], Mo et al., offered a sliding mode controller for guaranteeing vehicle stability after tire blowout with differential braking method based on improved vehicle system model. However, because of the linear sliding mode surface used, system states in traditional SMC scheme cannot converge to the equilibrium point in finite time. Lately, TSMC, employing the fractional power term instead of linear term in sliding mode surface, has been increasingly developed. By suitably designing the parameters, the TSMC assures the system states to reach the equilibrium point in finite time, and also offers some superior properties such as rapid 
response, strong robustness, and higher precision, see e.g., [31, 32, 33]. To this end, this scenario utilizes TSMC for accomplishing velocity tracking controller design motivated by the mentioned superiorities.

Inevitably, the tire blow-out vehicle will suffer from the various uncertainties, such as tire nonlinearities, unmodeled dynamics and external disturbances, which would destroy intrinsic performance of the controller. Identification and compensation of the uncertainty, as a kind of adaptive control, has been increasingly used in high-performance motion control of the complex electromechanical systems, such as motor control [34], robot manipulator [35, 36], mobile robot [37], and so on. Therefore, such adaptive design is here introduced for strengthening adaptability and robustness of the coordinated saturation planning and TSMC tracking control scheme. As well, due to its powerful approaching capacity, high convergence rate and superior approaching precision, radical basis function neural network ( $\operatorname{RBFNN}$ ) technique $[38,39,40]$ is utilized for identifying lumped uncertainties including of both sudden change and gradual change uncertainties.

\subsection{Contributions}

In light of the remarkable benefits and limitations, this study presents a stability control scheme for the vehicle with tire blowout based on saturated velocity planner and TSMC tracking controller combined with RBFNN approximator. Compared to the existing stability control methods for the vehicle with tire blowout, the contribution of this paper can be marked as the following significant points.

1. Saturated velocity planner is presented for maintaining the original trajectory in presence of longitudinal velocity and yaw rate saturation constraints, in which rear-end collision avoidance and vehicle system stability are enhanced simultaneously.

2. TSMC tracking controller is proposed for tracking the planned velocity signals in front of tire nonlinearities, unmodeled dynamics and external disturbances, where the lumped uncertainties are identified and compensated via RBFNN technique in real-time.

3. A composite control scheme is developed for the vehicle after tire blowout on expressway, in which system stabilization and original path tracking capacity are efficiently achieved by coordinating planning, tracking and approximation techniques. 


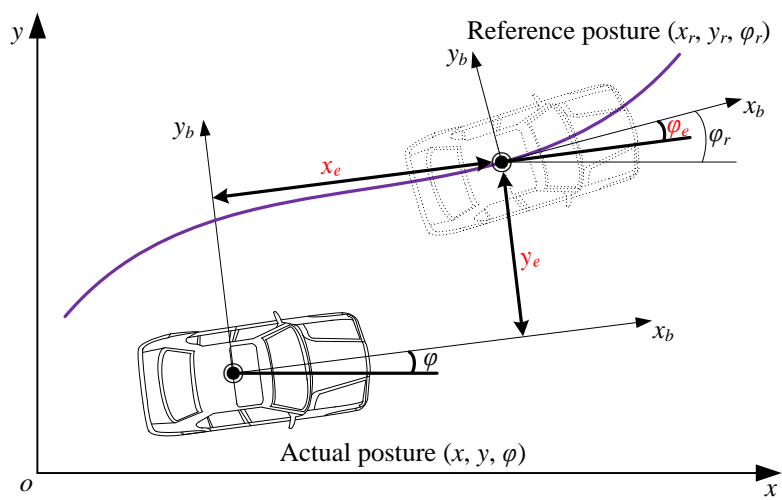

Figure 1: Posture errors relationship between actual and reference vehicles

The rest of this paper is organized as follows. Problem formulation is presented in Section 2, followed that saturated velocity planner and RBFNN based TSMC tracking controller are designed in Section 3. Simulation results and discussions are provided in Section 4, and conclusions are drawn in Section 5.

\section{Problem Formulation}

Consider the motion trajectory of the vehicle in inertia coordinate, as illustrated in Fig.1, the tracking errors in vehicle-fixed coordinate can be expressed as

$$
\left[\begin{array}{l}
x_{e} \\
y_{e} \\
\varphi_{e}
\end{array}\right]=\left[\begin{array}{ccc}
\cos \varphi & \sin \varphi & 0 \\
-\sin \varphi & \cos \varphi & 0 \\
0 & 0 & 1
\end{array}\right]\left[\begin{array}{l}
x_{r}-x \\
y_{r}-y \\
\varphi_{r}-\varphi
\end{array}\right]
$$

where $x_{e}, y_{e}, \varphi_{e}$ are the longitudinal, lateral and angle errors, respectively; $\left(x_{r}, y_{r}, \varphi_{r}\right)$ and $(x, y, \varphi)$ are the reference and actual postures of the vehicle in inertia coordinate, and their differential forms with respect to time are separately governed by

$$
\left\{\begin{array}{l}
\dot{x}_{(r)}=v_{(r)} \cos \varphi_{(r)} \\
\dot{y}_{(r)}=v_{(r)} \sin \varphi_{(r)} \\
\dot{\varphi}_{(r)}=\omega_{(r)}
\end{array}\right.
$$


where $x, x_{r}$ are the actual and reference longitudinal displacements, $y, y_{r}$ are the actual and reference lateral displacements, and $\varphi, \varphi_{r}$ are the actual and reference yaw angles. Together with (2), differentiating (1) along with the time, it holds that

$$
\left\{\begin{array}{l}
\dot{x}_{e}=\omega y_{e}-v+v_{r} \cos \varphi_{e} \\
\dot{y}_{e}=-\omega x_{e}+v_{r}+v_{r} \sin \varphi_{e} \\
\dot{\varphi}_{e}=\omega_{r}-\omega
\end{array}\right.
$$

where $v_{r}$ and $\omega_{r}$ are the reference forward velocity and yaw rate, respectively, and $v$ and $\omega$ are the actual velocity and yaw rate. Therefore, the control problem can be translated into the velocity control law design issue, that is, to find the control signals as the following form

$$
v=v^{*}(t, \varphi, x, y), \omega=\omega^{*}(t, \varphi, x, y)
$$

such that $x(t) \rightarrow x_{r}(t), y(t) \rightarrow y_{r}(t)$ and $\varphi(t) \rightarrow \varphi_{r}(t)$ as time $t \rightarrow+\infty$ while guaranteeing the following constraints:

$$
|v(t)| \leq v_{\max }, \omega(t) \leq \omega_{\max } \quad \forall t \geq 0
$$

where $\omega_{\max }>\sup _{t \geq 0}\left|\omega_{r}(t)\right|$ and $v_{\max }>\sup _{t \geq 0}\left|v_{r}(t)\right|$ are given arbitrary positive constants. Whereas, these control signals are regarded as the desired ones for the next stage, thus it is defined as velocity planning module in this study, and correspondingly the next stage is looked upon as the velocity tracking module.

Ignoring roll, pitch and vertical behaviors, the dynamics of the vehicle in longitudinal, lateral and yaw plane can be formulated by

$$
\left\{\begin{array}{l}
m\left(\dot{v}_{x}-v_{y} \omega\right)=F_{X}-C_{a} v_{x}^{2} \\
m\left(\dot{v}_{y}+v_{x} \omega\right)=F_{Y} \\
I_{z} \dot{\omega}=M_{Z}
\end{array}\right.
$$

where $v_{x}$ and $v_{y}$ are the longitudinal and lateral velocities, respectively, $m$ is the mass of the vehicle, $I_{z}$ is the inertia moment of the vehicle, and $C_{a}$ is the aerodynamic drag coefficient. $F_{X}$, 
$F_{Y}, M_{Z}$ are the resultant longitudinal, lateral forces and yaw moment, which can be governed by

$$
\left\{\begin{aligned}
F_{X}= & \left(F_{x f l}+F_{x f r}\right) \cos \delta_{f}-\left(F_{y f l}+F_{y f r}\right) \sin \delta_{f} \\
& +F_{x r l}+F_{x r r} \\
F_{Y}= & \left(F_{y f l}+F_{y f r}\right) \cos \delta_{f}+\left(F_{x f l}+F_{x f r}\right) \sin \delta_{f} \\
& +F_{y r l}+F_{y r r} \\
M_{Z}= & l_{f}\left(F_{y f l}+F_{y f r}\right) \cos \delta_{f}+l_{s}\left(-F_{x r l}+F_{x r r}\right) \\
& +l_{f}\left(F_{x r l}+F_{x r r}\right) \sin \delta_{f}-l_{r}\left(F_{y r l}+F_{y r r}\right) \\
& +l_{s}\left(F_{x f r} \cos \delta_{f}-F_{y f r} \sin \delta_{f}\right) \\
& +l_{s}\left(F_{y f l} \sin \delta_{f}-F_{x f l} \cos \delta_{f}\right)
\end{aligned}\right.
$$

where $F_{x i}$ and $F_{y i}$ are the tire longitudinal and lateral forces, respectively, $i=f l, f r, r l, r r$ denotes the front-left, front-right, rear-left, rear-right wheels, $l_{f}$ and $l_{r}$ are the distances from center of gravity to front and rear axles, $l_{s}$ is half width of wheel base, and $\delta_{f}$ is the front-wheel steering angle. Considering the small front-wheel steering angle assumption, resultant force/moment (7) can be rewritten as

$$
\left\{\begin{aligned}
F_{X}= & \left(F_{x f l}+F_{x f r}\right) \cos \delta_{f}+F_{x r l}+F_{x r r} \\
F_{Y}= & F_{y f l}+F_{y f r}+F_{y r l}+F_{y r r} \\
M_{Z}= & l_{s}\left(F_{x f r}-F_{x f l}\right) \cos \delta_{f}-l_{r}\left(F_{y r l}+F_{y r r}\right) \\
& +l_{f}\left(F_{y f l}+F_{y f r}\right)+l_{s}\left(-F_{x r l}+F_{x r r}\right)
\end{aligned}\right.
$$

Herein, the complicated tire models are not preferred in controller design stage due to the requirements of the quick reaction and computation burden. Therefore, tire lateral forces at front and rear axles are approximated by $F_{y f}=c_{f} \alpha_{f}$ and $F_{y r}=c_{f} \alpha_{r}$ [41], with $\alpha_{f}$ and $\alpha_{r}$ being the tire front and rear sideslip angles formulated by

$$
\alpha_{f}=\delta_{f}-\frac{v_{y}+\omega l_{f}}{v_{x}}, \alpha_{r}=\frac{\omega l_{r}-v_{y}}{v_{x}} .
$$


At the same time, define the following virtual control inputs as

$$
\left\{\begin{array}{l}
u_{1}=F_{x f l} \cos \delta_{f}+F_{x r l} \\
u_{2}=F_{x f r} \cos \delta_{f}+F_{x r r} \\
u_{3}=\delta_{f}
\end{array}\right.
$$

Integrating with (8)-(10), the dynamics formulation (6) can be reexpressed as

$$
\left\{\begin{aligned}
\dot{v}_{x} & =v_{y} \omega+\frac{1}{m}\left(u_{1}+u_{2}\right)-\frac{1}{m} C_{a} v_{x}^{2}+\xi_{1} \\
\dot{v}_{y} & =-v_{x} \omega+\frac{c_{r} l_{r}-c_{f} l_{f}}{m v_{x}} \omega-\frac{c_{f}+c_{r}}{m v_{x}} v_{y}+\frac{c_{f}}{m} u_{3}+\xi_{2} \\
\dot{\omega} & =-\frac{c_{f} l_{f}^{2}+c_{r} l_{r}^{2}}{I_{z} v_{x}} \omega+\frac{c_{r} l_{r}-c_{f} l_{f}}{I_{z} v_{x}} v_{y}+\frac{c_{f} l_{f}}{I_{z}} u_{3} \\
& +\frac{l_{s}}{I_{z}}\left(u_{2}-u_{1}\right)+\xi_{3}
\end{aligned}\right.
$$

where $\xi_{1}, \xi_{2}, \xi_{3}$ are the unmodeled dynamics. Further, the dynamics formulation (11) can be expressed as

$$
\left\{\begin{array}{l}
\dot{v}_{x}=v_{y} \omega+\frac{1}{m}\left(u_{1}+u_{2}\right)+f_{1}\left(v_{x}, v_{y}, \omega\right) \\
\dot{v}_{y}=-v_{x} \omega+\frac{c_{f}}{m} u_{3}+f_{2}\left(v_{x}, v_{y}, \omega\right) \\
\dot{\omega}=\frac{c_{f} l_{f}}{I_{z}} u_{3}+\frac{l_{s}}{I_{z}}\left(u_{2}-u_{1}\right)+f_{3}\left(v_{x}, v_{y}, \omega\right)
\end{array}\right.
$$

with

$$
\left\{\begin{array}{l}
f_{1}\left(v_{x}, v_{y}, \omega\right)=-\frac{1}{m} C_{a} v_{x}^{2}+\xi_{1} \\
f_{2}\left(v_{x}, v_{y}, \omega\right)=\frac{c_{r} l_{r}-c_{f} l_{f}}{m v_{x}} \omega-\frac{c_{f}+c_{r}}{m v_{x}} v_{y}+\xi_{2} \\
f_{3}\left(v_{x}, v_{y}, \omega\right)=\frac{\left(c_{r} l_{r}-c_{f} l_{f}\right) v_{y}-\left(c_{f} l_{f}^{2}+c_{r} l_{r}^{2}\right) \omega}{I_{z} v_{x}}+\xi_{3}
\end{array}\right.
$$

where $f_{1,2,3}\left(v_{x}, v_{y}, \omega\right)$ are the lumped uncertainties acted on the longitudinal, lateral and yaw motions, respectively, which include of tire nonlinearities, unmodeled dynamics and external 
disturbances. To sum up, vehicle system dynamics with tire blowout can be governed by

$$
\dot{v}=\phi(v) v+B u+f
$$

where $\boldsymbol{v}=\left[v_{x}, v_{y}, \omega\right]^{T}$ represents the velocity vector, $\boldsymbol{f}=\left[f_{1}\left(v_{x}, v_{y}, \omega\right), f_{2}\left(v_{x}, v_{y}, \omega\right), f_{3}\left(v_{x}, v_{y}, \omega\right)\right]^{T}$ stands for the lumped uncertainty vector, $\boldsymbol{u}=\left[u_{1}, u_{2}, u_{3}\right]^{T}$ indicates the virtual control input vector, and the other matrices are given by

$$
\phi(v)=\left[\begin{array}{ccc}
0 & \omega & 0 \\
-\omega & 0 & 0 \\
0 & 0 & 0
\end{array}\right], \boldsymbol{B}=\left[\begin{array}{ccc}
\frac{1}{m} & \frac{1}{m} & 0 \\
0 & 0 & \frac{c_{f}}{m} \\
-\frac{l_{s}}{I z} & \frac{l_{s}}{I z} & \frac{c_{f} l_{f}}{I z}
\end{array}\right] .
$$

To sum up, the stability control issue of the vehicle after tire blowout can be accomplished in two steps, the fist step is to find the proper $v$ and $\omega$ for tracking the original trajectory in the presence of longitudinal velocity and yaw rate saturation constraints. While, the second is to design the proper front-wheel steering angle and virtual force signals in finite time under tire nonlinearities, unmodeled dynamics and external disturbances.

\section{Control System Synthesis}

To prohibit the injury caused by tire blowout, this study presents a novel stability control scheme for the vehicle with tire blowout by hierarchically designing the planner, controller and approximator, as illustrated in Fig.2.

Specifically, the saturated velocity planner, based on hyperbolic tangent function, is constructed for eliminating tracking errors so as to enhance vehicle driving stability under the longitudinal velocity and yaw rate saturation constraints. After that, the desired forward velocity and yaw rate are produced; together with the desired lateral velocity, all the desired velocity signals are delivered into the tracking control module, where the TSMC technique is utilized for designing the tracking controller. With the aid of TSMC, the velocity tracking errors can convergent to the equilibrium point in finite time, and the adverse dynamic behavior of the vehicle after tire blowout can be timely suppressed. Accordingly, the expected resultant tire force and front-wheel steering angle are generated, which are sent into the vehicle system model and forms the closed-loop control system, more details about the system model and dynamics can 


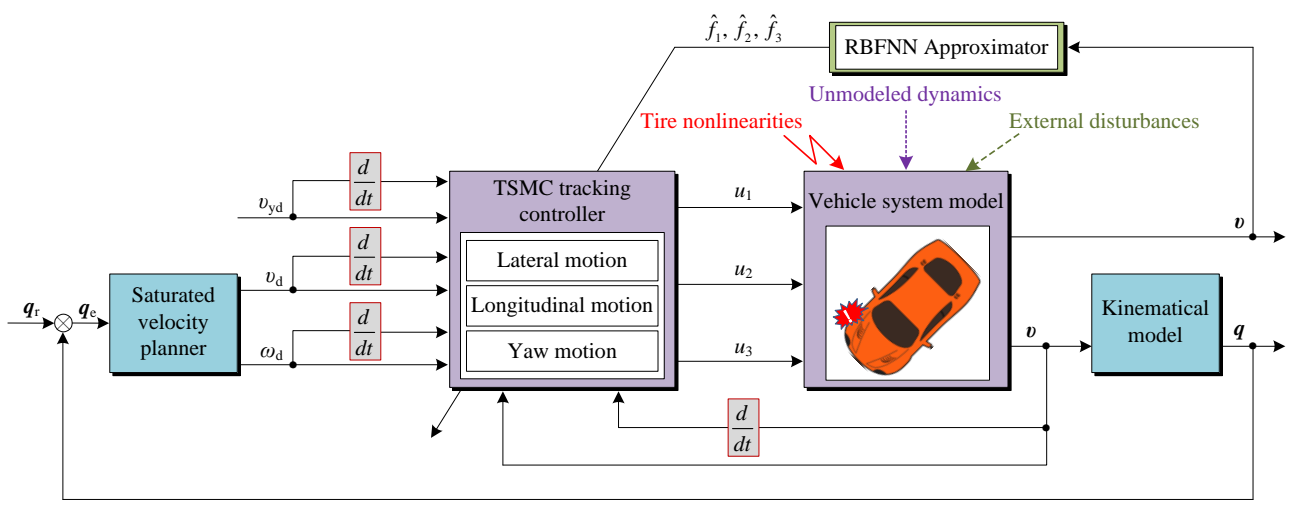

Figure 2: Stability control architecture for the vehicle with tire blowout on expressway

be found in [42]. In addition, a RBFNN approximator is proposed for approaching the lumped uncertainties to improve the adaptability capacity of the offered scheme.

The presented stability control scheme is a hierarchically closed-loop control architecture, which can considerably reduce the complexity of the controller and improve the robustness of control scheme.

\subsection{Saturated Velocity Planner}

Limited by longitudinal inter-vehicular space and system stability margin constraints, the forward velocity and yaw rate of the vehicle after tire blowout are usually constrained within a specified level. For this purpose, the saturated velocity planner is constructed for tracking the commanded trajectory, as follows

$$
\left\{\begin{array}{l}
v_{d}=v_{r} \cos \varphi_{e}+\lambda_{1} \tanh \left(x_{e}\right) \\
\omega_{d}=\omega_{r}+\frac{\lambda_{2} v_{r} y_{e}}{1+x_{e}^{2}+y_{e}^{2}} \frac{\sin \varphi_{e}}{\varphi_{e}}+\lambda_{3} \tanh \left(\varphi_{e}\right)
\end{array}\right.
$$

where $v_{d}$ and $\omega_{d}$ are desired forward velocity and yaw rate, respectively, $\lambda_{1}, \lambda_{2}$ and $\lambda_{3}$ are the positive design parameters.

Proposition 1. [9] Assume that $v_{r}$ and $\omega_{r}$ are bounded and uniformly continuous over $[0, \infty]$. If either $v_{r}(t)$ or $\omega_{r}(t)$ does not converge to zero, then the nonlinear dynamic error system (3) is globally asymptotically stable with the planner (16). 
Proof. Consider the following Lyapunov candidate function as

$$
V_{e}=\frac{\lambda_{2}}{2} \log \left(1+x_{e}^{2}+y_{e}^{2}\right)+\frac{1}{2} \varphi_{e}^{2}
$$

Differentiating (17) with respect to time, it results in

$$
\dot{V}_{e}=\frac{\lambda_{2}}{2} \frac{2 x_{e} \dot{x}_{e}+2 y_{e} \dot{y}_{e}}{1+x_{e}^{2}+y_{e}^{2}}+\varphi_{e} \dot{\varphi}_{e}
$$

Submitting (16) into (18), it holds that

$$
\begin{aligned}
\dot{V}_{e}= & \frac{\lambda_{2}\left(x_{e}\left(v_{r} \cos \varphi_{e}+\omega_{d} y_{e}-v_{d}\right)+y_{e}\left(v_{r} \sin \varphi_{e}-\omega_{d} x_{e}\right)\right)}{1+x_{e}^{2}+y_{e}^{2}} \\
& +\varphi_{e}\left(\omega_{r}-\omega_{d}\right) \\
= & \frac{-\lambda_{1} \lambda_{2}}{1+x_{e}^{2}+y_{e}^{2}} x_{e} \tanh \left(x_{e}\right)-\lambda_{3} \varphi_{e} \tanh \left(\varphi_{e}\right)
\end{aligned}
$$

It explicitly observes that $\exists h(x)=x \tanh (x)$ such that $h(x) \geq 0$ for $\forall x \in \mathbb{R}$, thereby deriving that $\dot{V}_{e} \leq 0$. The proof is completed.

In addition, because $-1 \leq \tanh (x) \leq 1$, it can be derived that $\left|\frac{y_{e}}{1+x_{e}^{2}+y_{e}^{2}}\right| \leq 1,\left|\frac{\sin \varphi_{e}}{\varphi_{e}}\right| \leq 1$. Further, it holds that

$$
\begin{aligned}
\left|\frac{\lambda_{2} v_{r} y_{e}}{1+x_{e}^{2}+y_{e}^{2}} \frac{\sin \varphi_{e}}{\varphi_{e}}\right| & =\left|\lambda_{2} v_{r}\right|\left|\frac{y_{e}}{1+x_{e}^{2}+y_{e}^{2}} \frac{\sin \varphi_{e}}{\varphi_{e}}\right| \\
& \leq\left|\lambda_{2} v_{r}\right|
\end{aligned}
$$

Therefore, the designed velocity planner is determined by

$$
\left\{\begin{array}{l}
\left|v_{d}\right| \leq\left|v_{r}\right|+\left|\lambda_{1}\right| \\
\left|\omega_{d}\right| \leq\left|\omega_{r}\right|+\left|\lambda_{2} v_{r}\right|+\left|\lambda_{3}\right|
\end{array}\right.
$$

It can be apparently seen that the boundaries of forward velocity and yaw rate are determined by the designed parameters $\lambda_{1}, \lambda_{2}$ and $\lambda_{3}$; the constraints of forward velocity and yaw rate can be readily handled through adjusting the three designed parameters. Besides, the proposed velocity planner can attenuate the system chattering phenomenon by introduced $\tanh (\cdot)$ function. Such improvement can strictly guarantee the continuity of the planned velocity signals compared to 
the simply use of the saturation element, which is more acceptable for stability control of the vehicle with tire blowout, this is also one of core contributions. Note that the desired longitudinal velocity $v_{x d}$ is equal to the desired velocity $v_{d}$ generated in the saturated velocity planner because the desired lateral velocity is zero for enhancing vehicle trajectory tracking performance.

\subsection{TSMC Tracking Controller}

Because of its inherent finite time convergence rate and superior steady-state property as stated previously, TSMC technique is utilized for designing velocity tracking controller, whose terminal sliding manifold is defined as follows

$$
\boldsymbol{s}=\dot{\boldsymbol{e}}+\boldsymbol{\kappa} E, \dot{E}=\operatorname{sig}^{\alpha}(\dot{\boldsymbol{e}})
$$

where $\boldsymbol{e}=\left[x_{d}-x, y_{d}-y, \varphi_{d}-\varphi\right]^{T}$ is the error vector in vehicle-fixed coordinate, $\boldsymbol{\kappa}=$ $\operatorname{diag}\left(\left[\kappa_{1}, \kappa_{2}, \kappa_{3}\right]\right)$ with $\kappa_{1}, \kappa_{2}, \kappa_{3}$ being positive design parameters, $\boldsymbol{s}=\left[s_{1}, s_{2}, s_{3}\right]^{T}, \operatorname{sig}^{\alpha}(\boldsymbol{e})=$ $\operatorname{sgn}(\boldsymbol{e})|\boldsymbol{e}|^{\alpha}$, and $\boldsymbol{\alpha}=\operatorname{diag}\left(\left[\alpha_{1}, \alpha_{2}, \alpha_{3}\right]\right)$ with $0<\alpha_{i}<1(i=1,2,3)$. Employ exponential reaching law as $\dot{\boldsymbol{s}}=-\boldsymbol{\sigma s}-\varrho \operatorname{sgn}(\boldsymbol{s})$, wherein $\boldsymbol{\sigma}=\operatorname{diag}\left(\left[\sigma_{1}, \sigma_{2}, \sigma_{3}\right]\right)$ and $\varrho=\operatorname{diag}\left(\left[\varrho_{1}, \varrho_{2}, \varrho_{3}\right]\right)$ with $\sigma_{i}(i=1,2,3)$ and $\varrho_{i}(i=1,2,3)$ being positive design parameters, respectively. Then, system control law can be designed as follows

$$
\boldsymbol{u}=\boldsymbol{B}^{-1}\left(\dot{\boldsymbol{v}}_{d}+\boldsymbol{\kappa} \dot{E}+\sigma s+\varrho \operatorname{sgn}(s)-\phi(v) v\right)
$$

where $\boldsymbol{v}_{d}=\left[v_{x d}, v_{y d}, \omega_{d}\right]^{T}$ is the desired velocity vector.

Theorem 1. For the system (12), if the terminal sliding manifold is chosen as (22), and the controller is designed as (23), then tracking error will converge to zero in a finite time.

Proof. Select the following Lyapunov candidate function as

$$
V_{1}=\frac{1}{2} s^{T} s
$$


Differentiating (24) with respect to time, it achieves that

$$
\begin{aligned}
\dot{V}_{1} & =\boldsymbol{s}^{T} \dot{\boldsymbol{s}}=\boldsymbol{s}^{T}(\ddot{\boldsymbol{e}}+\boldsymbol{\kappa} \dot{E}) \\
& =\boldsymbol{s}^{T}\left(\dot{\boldsymbol{v}}_{d}-\dot{\boldsymbol{v}}+\boldsymbol{\kappa} \dot{E}\right) \\
& =\boldsymbol{s}^{T}\left(\dot{\boldsymbol{v}}_{d}-\dot{\boldsymbol{v}}+\boldsymbol{\kappa} \dot{E}\right) \\
& =\boldsymbol{s}^{T}\left(\dot{\boldsymbol{v}}_{d}-(\boldsymbol{\phi}(\boldsymbol{v}) \boldsymbol{v}+\boldsymbol{B u})+\boldsymbol{\kappa} \dot{E}\right)
\end{aligned}
$$

Submitting control law (23) into (25), it derives that

$$
\dot{V}_{1}=s^{T}(-\sigma s-\varrho \operatorname{sgn}(s)) \leq 0
$$

Then, the Theorem 1 can be proven in the sense of Lyapunov stability theorem.

Notice that all the signals in the closed-loop system are bounded and the convergence time can be adjusted by selecting appropriate design parameters $\boldsymbol{\kappa}$ and $\boldsymbol{\alpha}$. In addition, according to the Lemma 1 in [32], the finite time $t_{s}$ can be governed by

$$
\begin{aligned}
t_{s} & =\left[\begin{array}{lll}
t_{s_{1}} & t_{s_{2}} & t_{s_{3}}
\end{array}\right]^{T} \\
& =\left[\begin{array}{lll}
\frac{\left|\dot{e}_{1}(0)\right|^{\left(1-\alpha_{1}\right)}}{\kappa_{1}\left(1-\alpha_{1}\right)} & \frac{\left|\dot{e}_{2}(0)\right|^{\left(1-\alpha_{2}\right)}}{\kappa_{2}\left(1-\alpha_{2}\right)} & \frac{\left|\dot{e}_{3}(0)\right|^{\left(1-\alpha_{3}\right)}}{\kappa_{3}\left(1-\alpha_{3}\right)}
\end{array}\right]^{T}
\end{aligned}
$$

where $t_{s_{1}}, t_{s_{2}}$ and $t_{s_{3}}$ denote the terminal times for the longitudinal, lateral and yaw motion subsystems, respectively.

\subsection{RBFNN based TSMC Tracking Controller}

In order to improve the adaptability and robustness of control scheme, the RBFNN approximator is developed for identifying the lump uncertainties since its powerful approaching capacity and superior approaching precision. The architecture of this approximator is demonstrated in Fig.3, from which it can be observed that RBFNN is a three-layered neural network structure, composed of input layer, hidden layer and output layer. Define $\boldsymbol{X}=\left[x_{1}, x_{2}, \cdots, x_{n}\right]^{T}$ as the input vector, then the radial basis function can be expressed as

$$
h_{j}(\boldsymbol{X})=\exp \left(-\frac{\left\|\boldsymbol{X}-\boldsymbol{c}_{j}\right\|^{2}}{2 b_{j}^{2}}\right)
$$




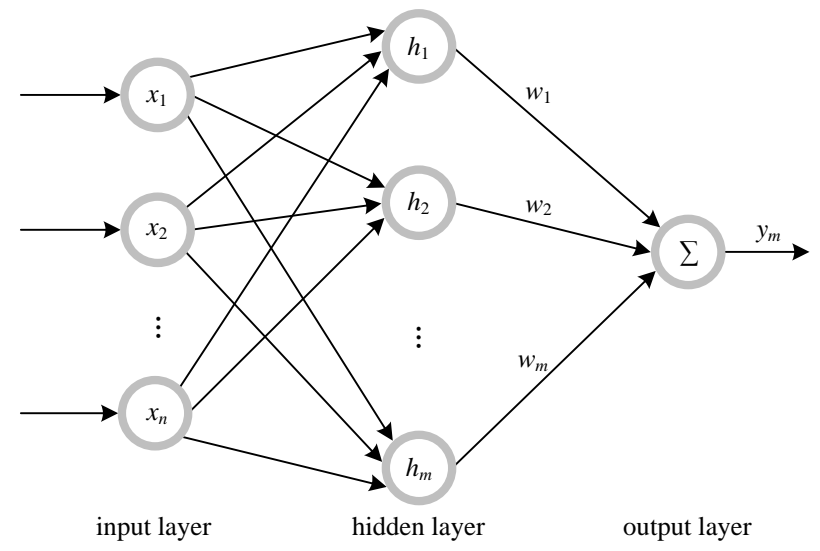

Figure 3: Architecture of RBFNN system

where $\boldsymbol{c}_{j}=\left[c_{j 1}, c_{j 2}, \cdots, c_{j n}\right]$ is the center value of neural net $j$, and $b_{j}$ is the width value of Gaussian function for neural net $j$, with $j$ being hidden neural net number in the hidden layer. Then, the output of the RBFNN can be acquired by

$$
\begin{aligned}
y_{m} & =w_{1} h_{1}+w_{2} h_{2}+\cdots+w_{m} h_{m} \\
& =\boldsymbol{W}^{T} \boldsymbol{h}(\boldsymbol{X})
\end{aligned}
$$

where $\boldsymbol{W}=\left[w_{1}, w_{2}, \cdots, w_{m}\right]^{T}$ is the weighting vector, and $\boldsymbol{h}(\boldsymbol{X})=\left[h_{1}, h_{1}, \cdots, h_{m}\right]^{T}$ is the radial basis vector. Define the identified error as $\tilde{f}=\hat{f}-\boldsymbol{f}$, and $\hat{f}$ is the identified lumped uncertainties. Since the slowly time-varying property of the lump uncertainties, it can be deduced that $\dot{\tilde{f}}=\dot{\hat{f}}$. Therefore, the identified lumped uncertainties can be governed by

$$
\hat{f}=\hat{\boldsymbol{W}}^{T} \boldsymbol{h}(\boldsymbol{X})
$$

with the weighting updating law as follows

$$
\dot{\hat{W}}=-\boldsymbol{\Omega h}(\boldsymbol{X}) \boldsymbol{s}^{T}
$$

where $\boldsymbol{\Omega}=\operatorname{diag}\left(\left[\Omega_{1}, \Omega_{2}, \Omega_{3}\right]\right)$, with $\Omega_{i}(i=1,2,3)$ being the positive design parameters.

$$
\boldsymbol{u}=\boldsymbol{B}^{-1}\left(\dot{\boldsymbol{v}}_{d}+\kappa \dot{E}+\sigma s+\varrho \operatorname{sgn}(s)-\phi(v) v-\hat{f}\right)
$$


Theorem 2. Considering the nonlinear dynamic system described by (12), if the trajectory tracking control law is designed as (32) with the identified uncertainties (30) and updating law (31), then all the tracking errors asymptotically converge to zero in finite time.

Proof. Define a Lyapunov candidate function as

$$
V=\frac{1}{2} \boldsymbol{s}^{T} \boldsymbol{s}+\frac{1}{2} \operatorname{tr}\left(\tilde{\boldsymbol{W}}^{T} \boldsymbol{\Omega}^{-1} \tilde{\boldsymbol{W}}\right)
$$

Differentiating (33) with respect to time yields that

$$
\begin{aligned}
\dot{V}= & \boldsymbol{s}^{T}(\hat{f}-\boldsymbol{f}-\sigma \boldsymbol{s}-\varrho \operatorname{sgn}(\boldsymbol{s}))+\operatorname{tr}\left(\tilde{\boldsymbol{W}}^{T} \Omega^{-1} \dot{\tilde{\boldsymbol{W}}}\right) \\
= & \boldsymbol{s}^{T}\left(\hat{\boldsymbol{W}}^{T} \boldsymbol{h}(\boldsymbol{X})-\boldsymbol{W}^{T} \boldsymbol{h}(\boldsymbol{X})-\sigma \boldsymbol{s}-\varrho \operatorname{sgn}(\boldsymbol{s})\right) \\
& +\operatorname{tr}\left(\tilde{\boldsymbol{W}}^{T} \Omega^{-1} \dot{\tilde{\boldsymbol{W}}}\right) \\
= & -\boldsymbol{s}^{T} \boldsymbol{\sigma} \boldsymbol{s}-\boldsymbol{s}^{T} \varrho \operatorname{sgn}(\boldsymbol{s})+\boldsymbol{s}^{T} \tilde{\boldsymbol{W}}^{T} \boldsymbol{h}(\boldsymbol{X}) \\
& +\operatorname{tr}\left(\tilde{\boldsymbol{W}}^{T} \boldsymbol{\Omega}^{-1} \dot{\hat{\boldsymbol{W}}}\right) \\
= & -\boldsymbol{s}^{T} \boldsymbol{\sigma} \boldsymbol{s}-\boldsymbol{s}^{T} \varrho \operatorname{sgn}(\boldsymbol{s})+\operatorname{tr}\left(\tilde{\boldsymbol{W}}^{T} \boldsymbol{h}(\boldsymbol{X}) \boldsymbol{s}^{T}\right) \\
& +\operatorname{tr}\left(\tilde{\boldsymbol{W}}^{T} \boldsymbol{\Omega}^{-1} \dot{\hat{\boldsymbol{W}}}\right)
\end{aligned}
$$

Substituting weighting updating law (31) into (34), it concludes that

$$
\begin{aligned}
\dot{V}= & -\boldsymbol{s}^{T} \boldsymbol{\sigma s}-\boldsymbol{s}^{T} \varrho \operatorname{sgn}(\boldsymbol{s})+\operatorname{tr}\left(\tilde{\boldsymbol{W}}^{T} \boldsymbol{h}(\boldsymbol{X}) \boldsymbol{s}^{T}\right) \\
& +\operatorname{tr}\left(\tilde{\boldsymbol{W}}^{T} \boldsymbol{\Omega}^{-1}\left(-\boldsymbol{\Omega} \boldsymbol{h}(\boldsymbol{X}) \boldsymbol{s}^{T}\right)\right) \\
= & -\boldsymbol{s}^{T} \boldsymbol{\sigma} \boldsymbol{s}-\boldsymbol{s}^{T} \varrho \operatorname{Sgn}(\boldsymbol{s})
\end{aligned}
$$

Obviously, $\dot{V} \leq 0$ is satisfied, and the Theorem 2 can be proven.

Therefore, the proposed velocity tracking controller can make the tracking errors converge to a compact set within a finite time via Lyapunov stability theorem, thereby guaranteeing the vehicle system stability in the presence of the lumped uncertainties. Compared with (23), control law (32) can considerably reduce the conservatism of the control method through the identification/compensation of RBFNN technique. 
Table 1: Nominal Vehicle Parameters

\begin{tabular}{lll}
\hline Parameters & Nominal Value & Unit \\
\hline$m$ & 1298.9 & $\mathrm{~kg}$ \\
$l_{f}$ & 1.000 & $\mathrm{~m}$ \\
$l_{r}$ & 1.454 & $\mathrm{~m}$ \\
$h_{g}$ & 0.533 & $\mathrm{~m}$ \\
$l_{s}$ & 1.436 & $\mathrm{~m}$ \\
$K_{s f}$ & 18900 & $\mathrm{Nm}$ \\
$I_{z}$ & 1627 & $\mathrm{~kg} \cdot \mathrm{m}^{2}$ \\
$c_{f}$ & 60000 & $\mathrm{~N} / \mathrm{rad}$ \\
$c_{r}$ & 60000 & $\mathrm{~N} / \mathrm{rad}$ \\
$R_{w}$ & 0.35 & $\mathrm{~m}$ \\
$R_{\text {blow }}$ & 0.25 & $\mathrm{~m}$ \\
$K_{s r}$ & 16800 & $\mathrm{Nm}$ \\
\hline
\end{tabular}

\section{Results and Discussions}

To validate the feasibility and effectiveness of the developed saturated velocity planning and tracking control scheme, the simulation studies for the vehicle subject to tire blowout on curved expressway, where system instability and fatal injury are much more likely to be triggered, are performed on the dry road surface with $\mu=0.8$ and $v_{r}=30 \mathrm{~m} / \mathrm{s}$. Due to space limitations, the frontright tire blowout scenario on the curved expressway with curvature $1 / 600 \mathrm{~m}^{-1}$ is only conducted for discussing the control performance of the designed control methods, where the structure parameters of the vehicle and Dugoff tire model are illustrated in Table.1, and the controller design parameters are indicated in Table.2. Finally, to evaluate the comprehensive performance, the tracking effect and control input of the TSMC and TSMC+RBFNN schemes are counted for discussions.

\subsection{Simulation Environment Setup}

Given the tremendous risk of tire blowout experiment, this study explores the system simulation platform based on the reported tire blowout experiment results, wherein the tire mechanical property variations $[43,44]$ are as follows: the rolling resistance coefficient will increase 30 times after tire blowout, the cornering stiffness will reduce to a quarter of the original value, and the radical stiffness will reduce to $1 / 15$ of the original value. Because the duration time is very short, the mechanical property variations are assumed linear during tire blowout. 
Table 2: Simulation Parameters for the Control System

\begin{tabular}{ll}
\hline & Design Parameters \\
\hline Planner & $\lambda_{1}=2, \lambda_{2}=0.1, \lambda_{3}=2$. \\
& $\kappa_{1}=\kappa_{2}=\kappa_{3}=0.5$, \\
& $\alpha_{1}=\alpha_{2}=\alpha_{3}=0.5$, \\
& $\sigma_{1}=2, \sigma_{2}=1, \sigma_{3}=2$, \\
& $\varrho_{1}=1, \varrho_{2}=2, \varrho_{3}=4$. \\
& $c_{1}=55[-1.5,-1,-0.5,0,0.5,1,1.5]$, \\
Approximator & $c_{2}=6[-1.5,-1,-0.5,0,0.5,1,1.5]$, \\
& $b_{j}=2, c_{2}=c_{3}, \Omega_{1}=1000$, \\
& $\Omega_{2}=200, \Omega_{3}=600$.
\end{tabular}

To imitate the lumped uncertainties coming from tire nonlinearities, unmodeled dynamics and external disturbances, the following uncertainties functions are constructed as

$$
\left\{\begin{array}{l}
\xi_{1}=\frac{\cos (t)}{2}-\frac{\sin (2 t)}{2}-5 \exp \left(\frac{(t-6)^{2}}{-2 p^{2}}\right)-\frac{F_{f}}{m} \\
\xi_{2}=\frac{\cos (t)}{10}-\frac{\sin (2 t)}{10}+2 \exp \left(\frac{(t-8)^{2}}{-2 p^{2}}\right) \\
\xi_{3}=\frac{\cos (2 t)}{10}-\frac{\sin (t)}{10}+3 \exp \left(\frac{(t-8)^{2}}{-2 p^{2}}\right)+\frac{M_{f}}{I_{z}}
\end{array}\right.
$$

where $p=0.5$ is standard variance, $F_{f}$ is the force uncertain term composed the wheel rolling resistance and aerodynamic drag resistance, and $M_{f}$ are the uncertain rolling resistance moment, both of which are formulated by

$$
\left\{\begin{array}{l}
F_{f}=k\left(F_{z f l}+F_{z f r}+F_{z r l}+F_{z r r}\right)+C_{a} v_{x}^{2} \\
M_{f}=l_{s}\left(k F_{z f l}-k F_{z f r}+k F_{z r l}-k F_{z r r}\right)
\end{array}\right.
$$

where $k$ is the rolling resistance coefficient, and $C_{a}$ is the aerodynamic drag coefficient. In particular, the resistance coefficient for the flat tire will change to 30 times of the original value as mentioned previously. Here, the nominal resistance coefficient employed in the simulation is 0.025 , the cornering stiffness of the flat tire is 25 percent of the original value, and the initial posture error is $\left[x_{e}, y_{e}, \varphi_{e}\right]^{T}=[0,0,0.0001]^{T}$. Focused on tire blowout on expressway, duration time of tire blowout is $0.1 \mathrm{~s}$, more details can refer to [24]. 
Considering the fact that the delay effect widely exists in the actuator and controller of vehicle system, the saturated velocity planner begin to be replanned with 0.1 s delay after blowout, and the TSMC controller start to track the desired velocity signals with $0.2 \mathrm{~s}$ delay based on the nominal flat tire parameters. In addition, to evaluate the comprehensive performance of the proposed control scheme, the trajectory tracking effect and virtual control input are separately extracted in TSMC and TSMC+RBFNN schemes based on the same simulation environment configuration.

\subsection{Blowout Simulation Results}

This section takes the front-right as example for validating the effectiveness of the proposed control methods, and the time responses of the vehicle motion trajectory are indicated in Fig.4. It finds that the vehicle motion trajectory can catch up with the reference trajectory in both the TSMC and TSMC+RBFNN schemes with the aid of the saturated velocity planner, both floating within the benchmark $x_{e}=1 \mathrm{~m}, y_{e}=0.5 \mathrm{~m}, \varphi_{e}=0.1 \mathrm{rad}$, which validates the effectiveness of the planner and controller. Further, details-oriented tracking errors of both schemes are plotted in Fig.5, in which the sums of absolute value of the tracking errors along with time are counted, and the results indicate that the tracking errors at each time in TSMC+RBFNN scheme are much smaller than that in TSMC one, thereby illustrating the efficiency of the composite control scheme. Due to the sharply increased rolling friction resistance after tire blowout, the longitudinal tracking error occupies a considerable proportion in the total tracking errors at each time. To sum up, the simulation results verify the feasibility of the stability control architecture of the vehicle with tire blowout, and comparative simulations highlights the outstanding robustness of TSMC+RBFNN scheme in enhancing original trajectory tracking capacity of the vehicle after tire blowout.

Time responses of the longitudinal velocity, lateral velocity and yaw rate are depicted in Figs.6-8, from which it observes that all the velocity signals have emerged violent oscillation after tire blowout when $t=6 \mathrm{~s}$. In Fig.6, the planed longitudinal velocity and actual longitudinal velocities in the two schemes are contrastively displayed, from which one can observes that the desired longitudinal velocity is rather small, thereby verifying the effectiveness of the developed saturated velocity planner. Moreover, with the aid of TSMC velocity tracking controller, the actual longitudinal velocities in both schemes can quickly track the desired one; however, due to the uncertainties in vehicle system, the longitudinal velocity in TSMC scheme emerges the large oscillation during tracking process. On the contrary, the TSMC+RBFNN scheme 


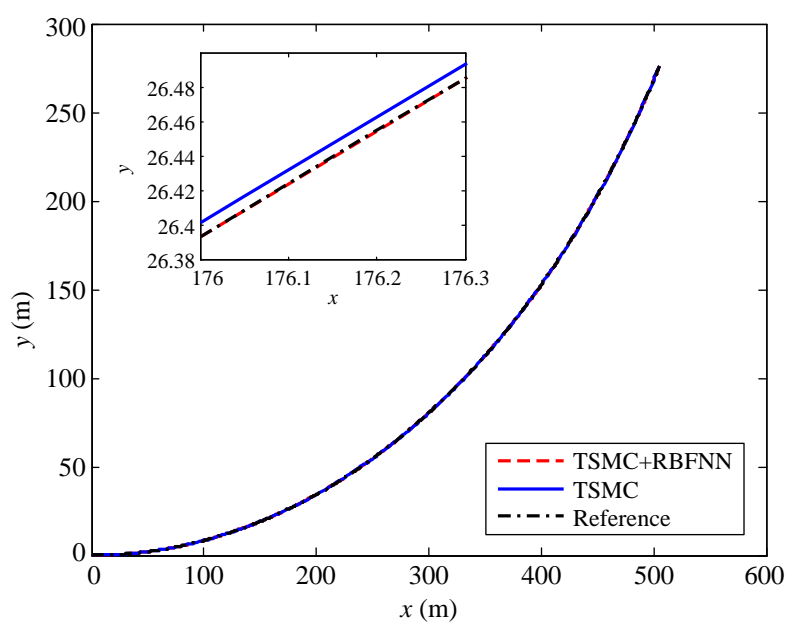

Figure 4: Time responses of the motion trajectory
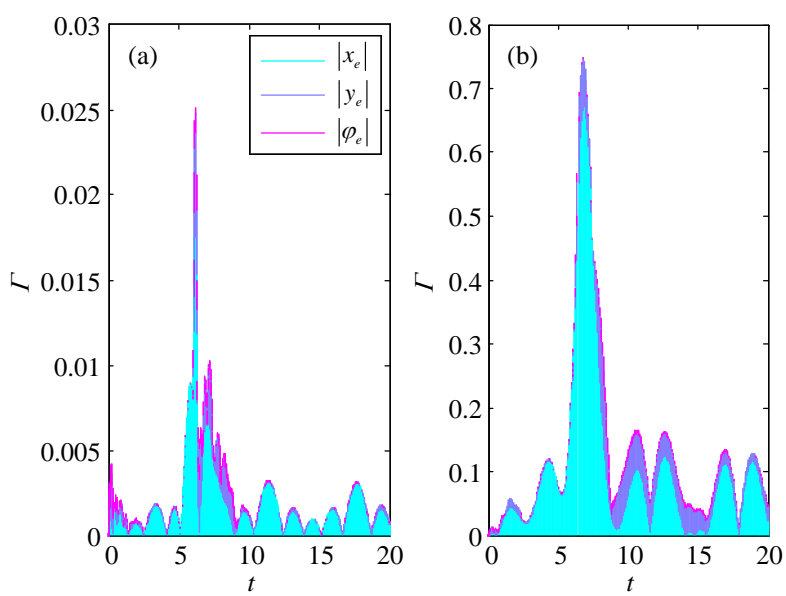

Figure 5: Time responses of the tracking errors: (a) TSMC+RBFNN, (b) TSMC 


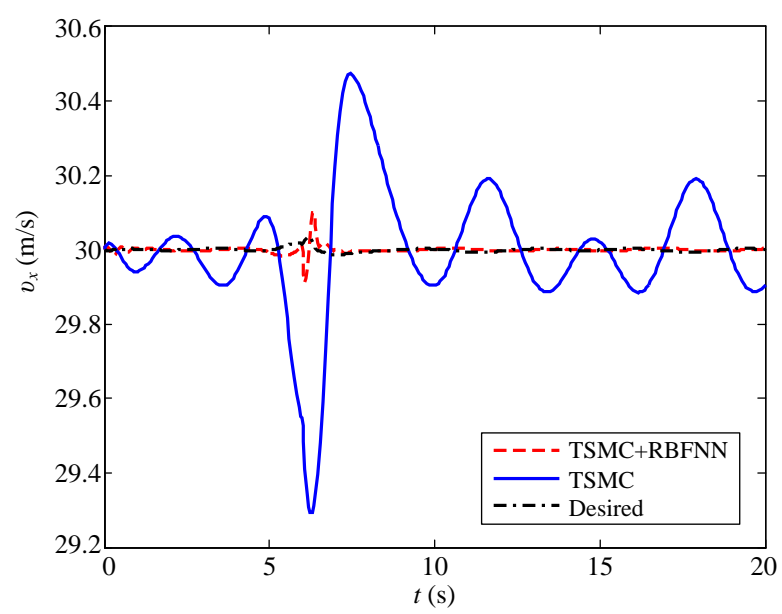

Figure 6: Time responses of the longitudinal velocity

provides a satisfactory tracking performance with the compensated lumped uncertainties, which demonstrates the robustness and adaptability of the proposed method. Meanwhile, the lateral velocity responses, as depicted in Fig.7, present the similar tendency to the longitudinal velocity responses; but, consider the vehicle is a nonholonomic system, the desired lateral velocity is thus zero. Lastly, the yaw rate responses of the vehicle, shown in Fig.8, appear the violent fluctuations in TSMC scheme during tracking process, especially after tire blowout, which significantly increases risk of system yaw instability; in contrast, the yaw rate responses in the TSMC+RBFNN scheme are milder, which thereby validates the efficiency and effectiveness of the adaptive scheme. To sum up, the desired velocities are reasonably planned subject to longitudinal velocity and yaw rate saturation constraints with the saturated velocity planner, and the developed tracking controller can efficiently follow the desired velocity signals in the presence of the lumped uncertainties.

Time responses of virtual control inputs are demonstrated in Fig.9, which exhibit the similar evolution law in both schemes. After front-right tire blowout, the rolling resistance of this wheel will sharply increased, which forces vehicle to drift into the flat tire side without active control. Thus, the control input $u_{2}$ begins to increase for assuring vehicle lateral stability, while the control input $u_{1}$ starts to decrease for balancing the control inputs of left and right sides of the vehicle. But, the control inputs in TSMC scheme undergo the drastic oscillation due to the tire nonlinearities, unmodeled dynamics and external disturbances; with the identification 


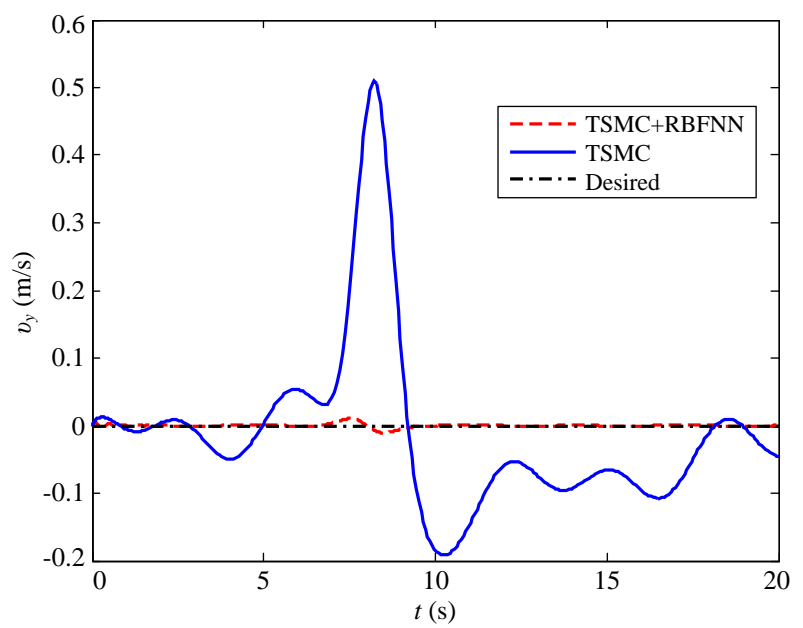

Figure 7: Time responses of the lateral velocity

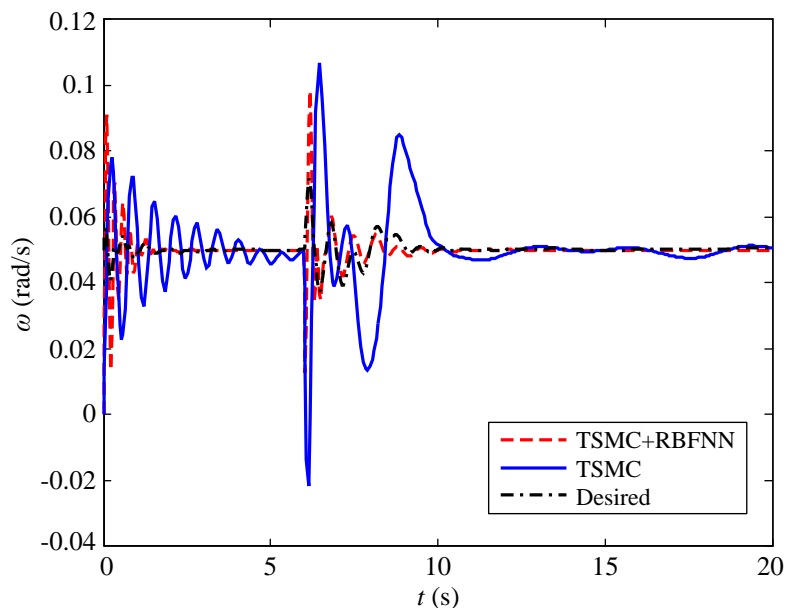

Figure 8: Time response of the yaw rate 

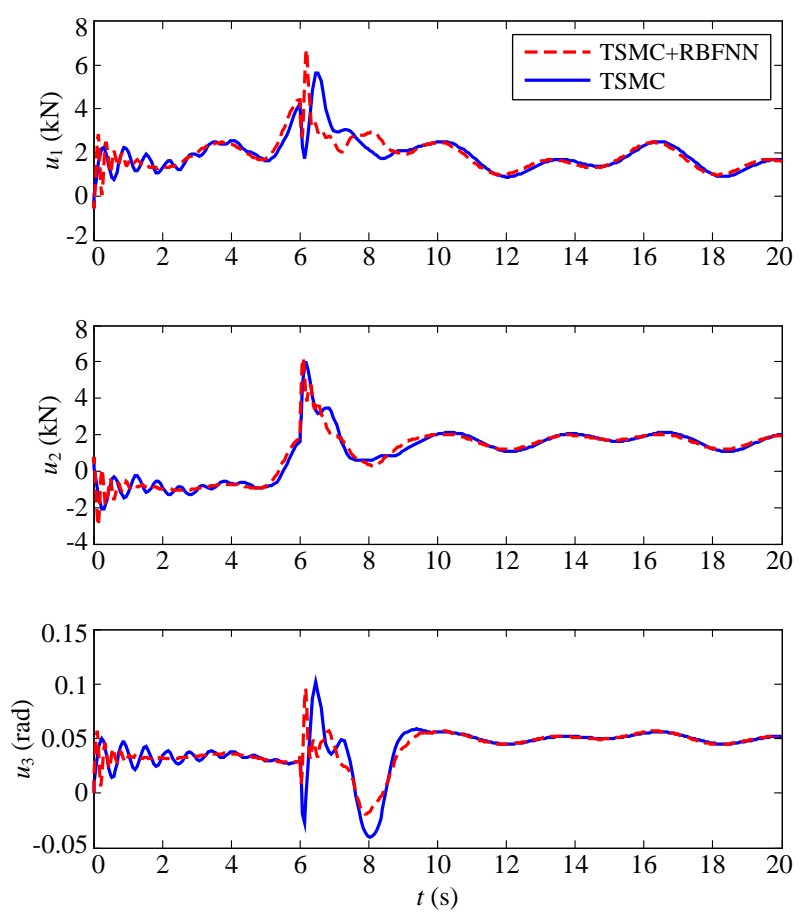

Figure 9: Time responses of virtual control inputs

and compensation of RBFNN, control inputs in TSMC+RBFNN scheme are relatively smooth. Because the tire blowout duration time is very short, control input $u_{1}$ has increased rapidly to maintain vehicle stability when tire blowout occurs; but, it has quickly dropped to a normal scope. In the absence of compensations, it needs much more front-wheel steering for the TSMC scheme to ensure vehicle stability after tire blowout. Summarily, the control inputs in both schemes are very near, implying that these consumes almost equal energy; but, with the identification and compensation of the RBFNN approximator, the control inputs in TSMC+RBFNN scheme is more stable and smoother, which thereby is preferred in the real-world application.

\subsection{Evaluations and Discussions}

To visually evaluate the comprehensive performance of the two control schemes, the root mean square error (RMSE) and root mean square (RMS) indices are separately introduced for discussing the reference trajectory tracking performance and virtual control input. The RMSE represents the deviation degree between the actual posture and reference posture in inertial 


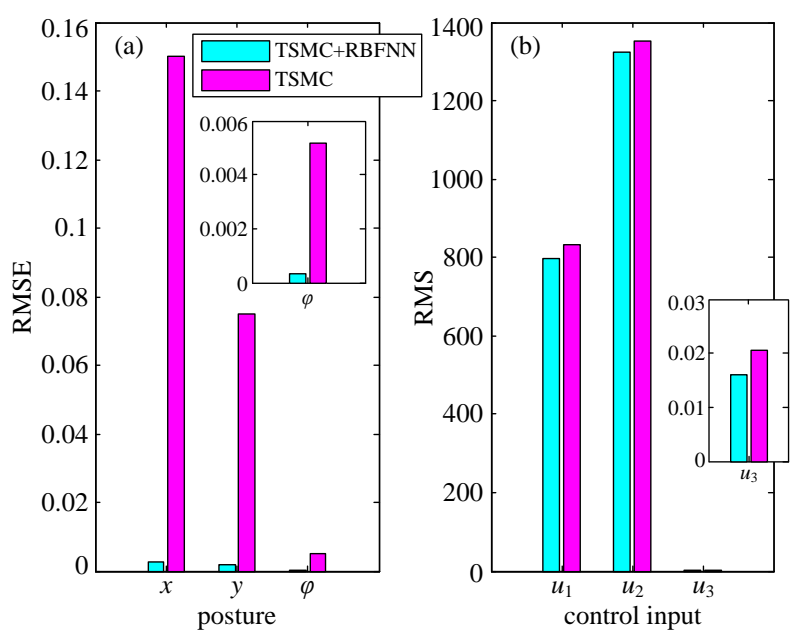

Figure 10: RMSE of the vehicle posture and RMS of the control input: (a) RMSE of the vehicle posture, (b) RMS of the control input

coordinate, which is governed by

$$
\operatorname{RMSE}(p)=\sqrt{\frac{\sum_{i=1}^{n}\left(p_{i}-p_{i, r}\right)^{2}}{n}}
$$

where $p_{i}=x_{i}, y_{i}, \varphi_{i}$ is the actual posture of the vehicle, $p_{i, r}=x_{i, r}, y_{i, r}, \varphi_{i, r}$ is the reference one, $n$ is the total sampling times, and $i$ is the $i$ th time. While, the RMS index reflects the dispersion degree of the control input, which is the root mean square between the actual control input and the mean value of the actual control input, formulated by

$$
\operatorname{RMS}(u)=\sqrt{\frac{\sum_{i=1}^{n}\left(u_{i}-\bar{u}_{i}\right)^{2}}{n}}
$$

where $u_{i}=u_{1}, u_{2}, u_{3}$ is the control input, $\bar{u}_{i}=\bar{u}_{1}, \bar{u}_{2}, \bar{u}_{3}$ is the mean value of control input. By (38) and (39), the RMSE of the trajectory and the RMS of the control input in both schemes are drawn in Fig.10. It finds that the RMSE of the posture in both scheme is quite small, which implies that both schemes can achieve the original trajectory tracking even if suffered from the lumped uncertainties, which can be demonstrated by Fig.10(a); but, the RMSE of the posture in longitudinal, lateral, yaw directions are greatly differ. Further, the RMS results of the control input are displayed in Fig.10(b), all the control input in both schemes are near; relatively, the 

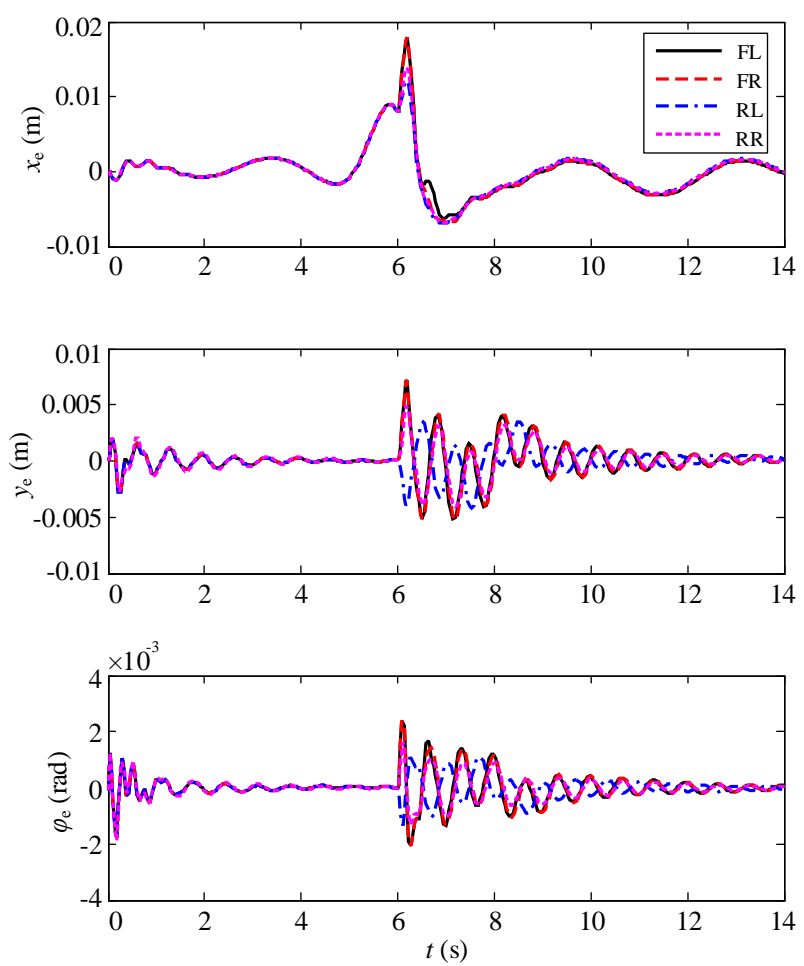

Figure 11: Trajectory tracking errors after different tire blowout, FL:front-left, FR:front-right, RL:rear-left, RR:rear-right

control inputs in TSMC varies more widely, this adds the risk of the tire bead separation from the rim. In addition, to further confirm the robustness of the proposed scheme, the original trajectory tracking effects of the vehicle after different tire blowout are presented in Fig.11, from which it observes that all the tracking errors are stabilized within the acceptable regions. Because these simulations are conducted on the same simulation environment and with the same controller parameters, in this sense, the robustness of the control scheme has been validated again. Note that the tracking errors are much smaller than the benchmarks, the benchmarks are therefore not marked in the figures.

To sum up, the excellent original trajectory tracking performance and vehicle dynamics responses are provided by the proposed control scheme, and the saturated velocity planning and TSMC method are successfully applied into the stability control of the vehicle with tire blowout. Besides, the RBFNN approximator improves the adaptability and robustness of the control scheme by identifying and compensating the lumped uncertainties. Evaluation results 
show that TSMC + RBFNN not only improve the original trajectory tracking, but also pose the considerable control input advantages. Therefore, the proposed control scheme provides an unified stability control framework for the vehicle with tire blowout by integrating velocity planning, tracking control, uncertainty identification and compensation. Since the developed controller relies critically on the estimated/measured velocity signals, thereby resulting in that the proposed method must carry tire blowout oriented estimator/sensor in fulfilling the predefined control tasks.

\section{Conclusion}

In this paper, a radial basis function neural network (RBFNN) based terminal sliding mode control (TSMC) scheme was proposed for electric ground vehicles after tire blowout on expressway in presence of tire nonlinearities, unmodeled dynamics and external disturbances. Firstly, forward velocity and yaw rate were effectively restrained within the expected scope via introduced hyperbolic tangent function in saturated velocity planner, where longitudinal velocity and yaw rate saturation constraints were handled successfully. Secondly, a TSMC velocity controller was designed to catch up with the planned velocity signals, where the RBFNN approximator was constructed to identify the lumped uncertainties. Finally, comparative simulation results demonstrated the effectiveness and efficiency of proposed adaptive control scheme in maintaining original trajectory tracking capacity and vehicle dynamics performance. In the near future, the hardware-in-the-loop (HiL) simulation experiment will be carried out in Lab to check and regulate the control law in the electronic control unit, and then the proposed control architecture and methods will be comprehensively evaluated.

\section{Acknowledgment}

This work was supported in part by the National Natural Science Foundation of China (Nos. 61873047 and 51975089), in part by the Major Research Plan of the National Natural Science Foundation of China (No. 91948203), in part by the Fundamental Research Funds for the Central Universities (DUT19ZD205), and in part by the State Key Laboratory of Robotics and System (HIT) under Grant SKLRS-2019-KF-17. 


\section{References}

[1] C. E. Beal, J. C. Gerdes, Model predictive control for vehicle stabilization at the limits of handling, IEEE Trans. Control Syst. Technol. 21 (2013) 1258-1269.

[2] D. J. Bu, X. Hu, Research on influencing factors and analysis of characteristics of vehicle tyre burst in traffic accident, in: Proceeding of the 16th International Forum of Automotive Traffic Safety (INFATS 2019), Hunan Univ., 2019, pp. 33-41.

[3] C. F. Hu, L. Cao, L. X. Zhao, N. Wang, Model predictive control-based steering control of unmanned ground vehicle with tire blowout, J. Tianjin Univ. (Sci. Technol.) 52 (2019) 468-474.

[4] S. Lu, M. J. Lian, Z. Cao, T. X. Zheng, Y. Xiao, X. Chen, Y. Ma, Active rectifying control of vehicle with tire blowout based on adaptive fuzzy proportional-integral-derivative control, Adv. Mech. Eng. 11 (2019) 1-13.

[5] W. Liu, X. W. Zhang, Study on fuzzy sliding mode control algorithm of vehicle stability control after a tire blowout, Mech. Sci. Technol. Aerosp. Eng. 38 (2019) 1944-1953.

[6] J.-S. Hu, Y. F. Wang, H. Fujimoto, Y. Hori, Robust yaw stability control for in-wheel motor electric vehicles, IEEE/ASME Trans. Mechatron. 22 (2017) 1360-1370.

[7] J. Velazquez Alcantar, F. Assadian, Vehicle dynamics control of an electric-all-wheel-drive hybrid electric vehicle using tyre force optimisation and allocation, Veh. Syst. Dyn. 57 (2019) 1897-1923.

[8] I. Han, Modelling the tyre forces for a simulation analysis of a vehicle accident reconstruction, Proc. Inst. Mech. Eng. Part D: J. Automob. Eng. 231 (2017) 16-26.

[9] Z. P. Jiang, E. Lefeber, H. Nijmeijer, Saturated stabilization and tracking of a nonholonomic mobile robot, Syst. Control Lett. 42 (2001) 327-332.

[10] H. Chen, C. Wang, B. Zhang, D. Zhang, Saturated tracking control for nonholonomic mobile robots with dynamic feedback, Trans. Inst. Meas. Control 35 (2013) 105-116.

[11] M. Van, S. S. Ge, H. L. Ren, Finite time fault tolerant control for robot manipulators using time delay estimation and continuous nonsingular fast terminal sliding mode control, IEEE Trans. Cybern. 47 (2016) 1681-1693.

[12] H. H. Pan, W. C. Sun, H. J. Gao, J. Y. Yu, Finite-time stabilization for vehicle active suspension systems with hard constraints, IEEE Trans. Intell. Transp. Syst. 16 (2015) 2663-2672.

[13] Q. L. Hu, X. D. Shao, Smooth finite-time fault-tolerant attitude tracking control for rigid spacecraft, Aerosp. Sci. Technol. 55 (2016) 144-157.

[14] H. Mirzaeinejad, Robust predictive control of wheel slip in antilock braking systems based on radial basis function neural network, Appl. Soft Comput. 70 (2018) 318-329.

[15] J. H. Guo, Y. G. Luo, K. Q. Li, Adaptive neural-network sliding mode cascade architecture of longitudinal tracking control for unmanned vehicles, Nonlinear Dyn. 87 (2017) 2497-2510.

[16] T. T. Gao, Y.-J. Liu, L. Liu, D. P. Li, Adaptive neural network-based control for a class of nonlinear pure-feedback systems with time-varying full state constraints, IEEE/CAA J. Autom. Sin. 5 (2018) 923-933.

[17] M. Bäcker, , A. Gallrein, , F. Calabrese, G. Leister, SIMULATION - simulation of a tire blow-out in a full vehicle scenario, in: 7th International Munich Chassis Symposium 2016, Springer, 2017, pp. 869-898.

[18] A. Sassi, S. Sassi, F. Tarlochan, Influence of tire blowout on the collision of a light pickup truck with a guardrail safety barrier, Proc. Inst. Mech. Eng. Part D: J. Automob. Eng. (2019) 1-11. 
[19] Y. Z. Cai, M. Y. Zang, Y. J. Chen, W. Liu, Experiments and finite element simulations of a tyre blow-out process, Proc. Inst. Mech. Eng. Part D: J. Automob. Eng. 228 (2014) 1116-1124.

[20] X. W. Zhang, F.-Y. Wang, Opportunities and challenges for tires intelligent manufacturing from intelligent tires, Sci. \& Technol. Rev. 36 (2018) 38-47.

[21] H. S. T. Patwardhan, Satyajit, M. Tomizuka, Experimental results of a tire-burst controller for AHS, Control Eng. Pract. 5 (1997) 1615-1622.

[22] D. F. Tandy, B. N. Ault, J. Colborn, R. Pascarella, Objective measurement of vehicle steering and handling performance when a tire loses its air, SAE Int. J. Passenger Cars: Mech. Syst. 6 (2013) 741-769.

[23] S. Patwardhan, M. Tomizuka, W. B. Zhang, P. Devlin, Theory and experiments of tire blow-out effects and hazard reduction control for automated vehicle lateral control system, in: American Control Conference, 1994, pp. 12071209.

[24] W. Blythe, T. D. Day, W. D. Grimes, 3-Dimensional simulation of vehicle response to tire blow-outs, 1998-26980221, 1998

[25] K. V. Chakravarthy, Development of a Steer Axle Tire Blowout Model for Tractor Semitrailers in TruckSim, Ph.D thesis, The Ohio State University, 2013.

[26] A. Li, Y. Chen, X. Y. Du, W.-C. Lin, Enhanced tire blowout modeling using vertical load redistribution and selfalignment torque, in: ASME 2019 Dynamic Systems and Control Conference, American Society of Mechanical Engineers Digital Collection, 2019.

[27] F. Wang, H. Chen, L. L. Guo, Y. F. Hu, Predictive safety control for road vehicles after a tire blowout, Sci. China Info. 61 (2018) 70209.

[28] H. H. Jing, Z. Y. Liu, Gain-scheduling robust control for a tire-blow-out road vehicle, Proc. Inst. Mech. Eng. Part D: J. Automob. Eng. 233 (2019) 344-362.

[29] Y. F. Cong, Motion planning and control for autonomous vehicle driving on motorways, Ph.D. dissertation. Jilin University, China. (2011).

[30] T. P. Mo, X. W. Zhang, K. F. Fan, W. Mo, Y. Q. Qiu, Design and simulation of the sliding mode controller for the vehicle blow-out process control, Int. J. Veh. Safety 6 (2013) 333-346.

[31] M. Chen, Q. X. Wu, R. X. Cui, Terminal sliding mode tracking control for a class of SISO uncertain nonlinear systems, ISA Trans. 52 (2013) 198-206.

[32] M. Yue, L. J. Wang, T. Ma, Neural network based terminal sliding mode control for wmrs affected by an augmented ground friction with slippage effect, IEEE/CAA J. Autom. Sin. 4 (2017) 498-506.

[33] Q. L. Hu, B. Y. Jiang, Continuous finite-time attitude control for rigid spacecraft based on angular velocity observer, IEEE Trans. Aerosp. Electron. Syst. 54 (2018) 1082-1092.

[34] A. Rodríguez-Molina, M. G. Villarreal-Cervantes, J. Álvarez-Gallegos, M. Aldape-Pé rez, Bio-inspired adaptive control strategy for the highly efficient speed regulation of the DC motor under parametric uncertainty, Appl. Soft Comput. 75 (2019) 29-45.

[35] C. G. Yang, Y. M. Jiang, W. He, J. Na, Z. J. Li, B. Xu, Adaptive parameter estimation and control design for robot manipulators with finite-time convergence, IEEE Trans. Ind. Electron. 65 (2018) 8112-8123.

[36] S. Zhang, Y. Dong, Y. C. Ouyang, Z. Yin, K. X. Peng, Adaptive neural control for robotic manipulators with output constraints and uncertainties, IEEE Trans. Neural Networks Learn. Syst. 29 (2018) 5554-5564. 
[37] W. He, Y. Dong, Adaptive fuzzy neural network control for a constrained robot using impedance learning, IEEE Trans. Neural Networks Learn. Syst. 29 (2017) 1174-1186.

[38] P. Kanirajan, V. S. Kumar, Power quality disturbance detection and classification using wavelet and RBFNN, Appl. Soft Comput. 35 (2015) 470-481.

[39] W. He, Y. K. Sun, Z. C. Yan, C. G. Yang, Z. J. Li, O. Kaynak, Disturbance observer-based neural network control of cooperative multiple manipulators with input saturation, IEEE Trans. Neural Networks Learn. Syst. (2019).

[40] H. Y. Li, L. Bai, L. J. Wang, Q. Zhou, H. Q. Wang, Adaptive neural control of uncertain nonstrict-feedback stochastic nonlinear systems with output constraint and unknown dead zone, IEEE Trans. Syst. Man Cybern. Part A Syst. 47 (2017) 2048-2059.

[41] H. Y. Guo, J. Liu, D. P. Cao, H. Chen, R. Yu, C. Lv, Dual-envelop-oriented moving horizon path tracking control for fully automated vehicles, Mechatronics 50 (2018) 422-433.

[42] L. Yang, M. Yue, H. Z. Zhang, G. Xu, Toward hazard reduction of road vehicle after tire blowout: A driver steering assist control strategy, in: 2019 38th Chinese Control Conference (CCC), IEEE, 2019, pp. 6600-6605.

[43] Y. L. Wang, K. H. Guo, Analysis of vehicles kinetic characteristic after tire blow-out, in: Advanced Materials Research, volume 455, Trans Tech Publ., 2012, pp. 1115-1121.

[44] H. Y. Guo, F. Wang, H. Chen, D. P. Guo, Stability control of vehicle with tire blowout using differential flatness based MPC method, in: Proceedings of the 10th World Congress on Intelligent Control and Automation, IEEE, 2012, pp. 2066-2071. 